\title{
Design of reliable communication networks
}

\author{
Eric Gourdin ${ }^{1}$ - Deep Medhi ${ }^{2} \cdot$ Achille Pattavina $^{3}$ \\ (C) Institut Mines-Télécom and Springer International Publishing AG, part of Springer Nature 2017
}

This special issue follows the 12th International Conference on the Design of Reliable Communication Networks (DRCN 2016) that was held in CNAM Paris, March 14-17, 2016. Since its creation in 1998, DRCN has become over the years a well-established forum for scientists of both industry and academy who have interest in reliability and availability of telecommunication networks, or other related topics on resilience.

The 2016 edition of DRCN was a very successful event with three keynotes, a tutorial, a panel on Network Disaster Management and Recovery, and plenary technical sessions addressing new research and application challenges regarding reliability and resilience.

The future $5 \mathrm{G}$ networks are the next step in the endless race for increased throughput requirements, bandwidth availabilities, and huge amounts of data to store or transfer every minute of every day and everywhere in the planet. In parallel, the recent rise of virtualization techniques, such as Network Function Virtualization (NFV) and a network softwarization paradigm, such as Software-Defined Networking (SDN), has started to change in depth the landscape of telecommunication. All these technological functionalities will offer huge possibilities to ease the deployment of new services or the dynamic management of network resources. As always, this gain in flexibility comes with a cost: since the combination of possibilities becomes almost endless, people in charge of defining new architectures or managing the future networks and

\section{Eric Gourdin}

Deep Medhi

DMedhi@umkc.edu

Achille Pattavina

achille.pattavina@polimi.it

Orange, France

2 University of Missouri, Kansas-City, MO, USA

3 Politecnico di Milano, Milan, Italy services will more than ever need sophisticated methods and tools to make the right decisions. In this context, ensuring that the networks are reliable, resilient, and able to survive or counter failures or attacks, is a very challenging issue. The research community has already invested this new trend and started to imagine solutions to handle the problems that will soon arise.

The papers in this special issue address some of these challenging new problems, trying to balance the new objectives with other types of objectives that are also nowadays identified as crucial for providing safety, containing attacks and disasters, and preserving the planet as much as possible. The papers cover a wide range of problems, from theoretical investigations of underlying graph structures to algorithmic guidelines and tools to address resiliency issues.

We received 19 submissions from an open call. After a thorough reviewing process where every paper has been evaluated by at least two experts, ten papers have been accepted for this special issue. Reviewers' comments were very helpful to select the most significant contributions as well as to improve the content, quality, and presentation of the accepted papers. Hereafter, a summary of each paper in this special issue is provided.

The first two papers address the fundamental problem of optimally designing resilient and efficient graph topologies. The resilience is based on the notion of paths disjointness and the efficiency relies on a path length limit.

- Ibrahima Diarrassouba, Meriem Mahjoub, A. Ridha Mahjoub, and Hande Yaman in their paper entitled "k Node-Disjoint Hop-Constrained Survivable Networks: Polyhedral Analysis and Branch \& Cut" investigate the problem of optimally designing topologies where there exists $\mathrm{k}$ node disjoint paths (each one of which of length less than or equal to L) for each given set of demands. Although similar, the problem is more restrictive (and in that sense, harder) than the problem with edge-disjoint requirements. The authors derive a ILP (Integer Linear Programming) model and analyze the associated polytope 
for the cases where $\mathrm{L}=2$ or 3 . They propose additional valid inequalities (also valid for the case $L=4$ ) and analyze the facial property of these inequalities. All these results are then embedded in a branch-and-cut algorithm whose performances are evaluated through extensive sets of numerical experiments.

- Quentin Botton, Bernard Fortz, and Luis Gouveia in their paper entitled "The 2 Edge-Disjoint 3-paths Polyhedron" study the problem of finding, within a given graph, a set of two edge disjoint paths, each of which of length less than or equal to 3 (counted in number of edges). A compact linear model is provided that is valid for general edge costs. The authors also provide a full description of the problem polyhedron.

The next paper addresses a joint content location and routing optimization problem:

- Dimitri Papadimitriou, Didier Colle, and Piet Demeester in their paper entitled "Mixed-Integer Optimization for the Combined capacitated Facility Location-Routing Problem" investigate a joint optimization problem where digital contents have to be located, and routing paths established so that sets of demands for the contents can be fulfilled. The problem displays non-linearities that need to be linearized so that a resolution approach can be applied. The joint modeling approach is compared to a sequential approach through series of numerical experiments.

The next two papers exploit a part of the multilayer structures on which most of the wide area networks are built, either to improve differentiated resiliency over two successive layers, or to leverage the use of sleep mode techniques in WDM networks to improve energy efficiency.

- Abdulaziz Alashaikh, David Tipper, and Teresa Gomes in their paper entitled "Exploring the Logical Layer to Support Differentiated Resilience Classes in Multilayer Networks" derive an approach where two logical layers with different availability requirements are mapped onto a physical network (with some restrictions on the layer with lower availability requirement). They propose a MILP (Mixed Integer Linear Programming) model to optimally design the two logical layers and define the routing paths of high availability within these layers. The lower priority demands are then routed within a second step according to a LP (Linear Programming) model. The approach is evaluated on four test networks from the literature.

- Ali Hmaity, Francesco Musumeci, and Massimo Tornatore in their paper entitled "Power Reduction Strategies with Differentiated Quality of Protection in IPover-WDM Networks" propose a trade-off between network efficiency and network resiliency. The approach focuses on WDM (Wavelength Division Multiplexing) using either sleep mode or an hourly scheduling optimized for energy efficiency. An ILP (Integer Linear Program) model based on edge-flow variables is proposed and evaluated over a 11 nodes and 26 bidirected links network instance. The analysis shows very significant gains in energy consumption.

The next paper addresses security issues and proposes to analyze the resiliency of an infrastructure against risk propagation with a new graph-based framework.

- M. Yassine Naghmouchi, Nancy Perrot, A. Ridha Mahjoub, Nizar Kheir, and Jean-Philippe Wary in their paper entitled "Assessing the Risk of Complex ICT Systems" propose a new risk assessment framework based on a graph (RAG or Risk Assessment Graph) modeling the potential propagations of risks between adjacent nodes, each node being a combination of an asset and a single vulnerability. The RAG can then be used together with various risk metrics to measure the potential impact of a given attack. This generic approach is then illustrated in the context of Software Defined Networks (SDN).

The last four papers propose approaches or tools to deal with geographically related disruptive events.

- Michael Todd Gardner, Yufei Cheng, Cory Beard, James P.G. Sterbenz, and Deep Medhi in their paper entitled "Provisioning Dynamic and Critical Demand Structures During Geographic Events Using Multi-Disaster Approach" propose a set of approaches to handle disaster events disrupting major parts of telecommunication networks. A MIP (Mixed-Integer Programming) model is proposed to depict a provisioning problem taking into account the fact that, during a disaster, the failures are geographically correlated. Another MIP is designed for critical service restoration, and several algorithms are proposed to solve these related problems.

- Sugang Xu, Noboru Yoshikane, Masaki Shiraiwa, Takehiro Tsuritani, Hiroaki Harai, Yoshinari Aawaji, and Naoya Wada in their paper entitled "Emergency Optical Network Planning with Multi-vendor Interconnection and Portable EDFAs" consider the problem of restoring connectivity within a geographic area where a disaster has occurred. The goal is then to optimally re-use surviving parts of several damaged interconnection networks and deploying some fiber amplifier equipment's (EFDAs) to extend the reach of optical links. The problem is modeled as a MILP (Mixed Integer Linear Programming) problem with a combination of several objectives. Some numerical tests are reported. 
- Lakshmi J. Mohan, Pablo Ignacio Serrano Caneleo, Udaya Parampalli, and Aaron Harwood in their paper entitled "Geo-Aware Erasure Coding for High Performance Erasure Coded Storage Clusters" investigate the benefits that could be achieved in the efficiency of distributed coded storage systems when some knowledge about the geographical location of the data centers is taken into account.

- Arun Das, Arunabha Sen, Chunming Qiao, Nasir Ghani, and Nathalie Mitton in their paper entitled "Raptor: A Network Tool for Mitigating the Impact of Spatially Correlated Failures in Infrastructure Networks" present a tool for analyzing the resilience of networks confronted to a set of simultaneous failures arising in a same geographical area. The tool implements a wide range of algorithms to compute region-based metrics and proposes several modules to analyze, among others, faults and paths.

Acknowledgments The guest editors would like to express their deep appreciation to the editor-in-chief, Prof. Guy Pujolle, for giving the opportunity to publish this special issue, as well as the journal editorial staff for their continuous support and guidelines during the process of this publication. The guest editors also thank all the authors for submitting quality articles and the reviewers for helping in the selection of papers and improving the accepted papers. 